\title{
Atypical antipsychotics-induced metabolic syndrome and nonalcoholic fatty liver disease: a critical review
}

This article was published in the following Dove Press journal:

Neuropsychiatric Disease and Treatment

\author{
Haiyun Xu \\ Xiaoyin Zhuang
}

The Mental Health Center, Shantou University Medical College, Shantou,

People's Republic of China
Correspondence: Haiyun Xu The Mental Health Center, Shantou University Medical College, Shantou 51504I, People's Republic of China Email hyxu@stu.edu.cn

\begin{abstract}
The atypical antipsychotics (AAPs) have been used as first-line drugs in psychiatric practice for a wide range of psychotic disorders, including schizophrenia and bipolar mania. While effectively exerting therapeutic effects on positive and negative symptoms, as well as cognitive impairments in schizophrenia patients, these drugs are less likely to induce extrapyramidal symptoms compared to typical antipsychotics. However, the increasing application of them has raised questions on their tolerability and adverse effects over the endocrine, metabolic, and cardiovascular axes. Specifically, AAPs are associated to different extents, with weight gain, metabolic syndrome (MetS), and nonalcoholic fatty liver disease (NAFLD). This article summarized clinical evidence showing the metabolic side effects of AAPs in patients with schizophrenia, and experimental evidence of AAPs-induced metabolic side effects observed in animals and cell culture studies. In addition, it discussed potential mechanisms involved in the APPs-induced MetS and NAFLD.
\end{abstract}

Keywords: antipsychotics, MetS, NAFLD, schizophrenia

\section{Introduction}

The introduction of chlorpromazine in 1952, a phenothiazine antipsychotic and dopamine inhibitor, initiated the pharmacological treatment of schizophrenia thus has been considered a revolutionary event in psychiatry. ${ }^{1}$ Several other antipsychotics have been produced since then, including fluphenazine, flupentixol, and haloperidol. These first-generation antipsychotics were named typical antipsychotics (TAPs) because of their main effects on dopamine D2 receptors. While the dopamine D2 receptors are being blocked, psychotic symptoms can be improved obviously. A major issue with TAPs is the limited ability to treat negative and cognitive domains of schizophrenia, alongside with the risk of extrapyramidal symptoms (EPS). ${ }^{2}$

Different from TAPs, which are primarily dopamine D2 antagonists, the atypical antipsychotics (AAPs) act at multiple neurotransmitter receptors, including dopamine D1, D2, D3, and D4, adrenergic alpha1 and alpha2, serotonin 5HT2A and 5HT2C, histamine and muscarinic receptors. Of the AAPs, clozapine, a dibenzodiazepine developed in 1961, is the first one approved for the treatment of resistant schizophrenia, ${ }^{3}$ but was withdrawn from the market in USA because it induced agranulocytosis in a patient. In 1980s, however, it was re-introduced for treatmentresistant patients. Following clozapine, risperidone was introduced in 1993, olanzapine in 1996, quetiapine in 1997, ziprasidone in 2001, aripiprazole in 2002, 
extended-release paliperidone in 2006, asenapine in 2009, iloperidone in 2009, and paliperidone long-acting injection in 2009. ${ }^{4}$

AAPs are now widely used to manage psychosis (including delusions or hallucinations, as well as disorders of thought), particularly in schizophrenia and bipolar disorder. Antipsychotics may also be used to counter psychosis associated with a wide range of other disorders, such as psychotic depression and obsessive-compulsive disorder. They became the most popular drug class in the US in $2008 .^{5}$ Because of their novel receptor binding profiles, AAPs have good efficacy regarding to negative symptoms (apathy, hypokinesis, poverty of thought, introverted thinking) and induce less EPS, which are more frequently seen in patients treated with TAPs and can impair patients' ability to complete activities of daily living. According to a recent large meta-analysis, the mean prevalence of tardive dyskinesia (TD) among current TAPs users was approximately $30 \%{ }^{6}$ Another meta-analysis reported that EPS was less frequent and severe in patients on several AAPs than their TAP comparators. Pooled AAPs produced significantly less EPS than TAPs. ${ }^{7}$

In addition to EPS, metabolic syndrome (MetS) in patients with schizophrenia has become a major concern as evidenced by the high levels of MetS in schizophrenia patients. According to a previous meta-analysis, almost 1 in 3 of unselected patients with schizophrenia meet criteria for MetS (see the definition described later), 1 in 2 patients are overweight, 1 in 5 appear to have significant hyperglycemia and at least 2 in 5 have lipid abnormalities. ${ }^{8}$ Although many other factors may contribute to the elevated prevalence rate of MetS in the schizophrenia population, several reviews and meta-analyses have demonstrated a role of certain AAPs. ${ }^{9-13}$ They cause variable degrees of metabolic side effects such as weight gain, obesity, dyslipidemia, insulin resistance, glucose intolerance, overt diabetes, nonalcoholic fatty liver disease (NAFLD) and, in rare cases, steatohepatitis. ${ }^{4,5}$ In this article, we will summarize clinical evidence for AAPs-induced metabolic side effects, include animal models analogous to clinical observations with AAPs-treated patients, and discuss the potential mechanisms involved in the AAPs-induced metabolic side effects.

\section{Mets and NAFLD}

MetS has been defined as a specific combination of metabolic risk factors, which may result in cumulative risk being greater than the sum of the individual risks. These risk factors include central obesity or body mass index, serum lipids, blood pressure, and serum glucose. ${ }^{4}$ They have been associated with the development of cardiovascular diseases (CVD) and excess mortality. ${ }^{14-16}$ As a prevalent condition and predictor of CVD, MetS provides the opportunity to identify high-risk populations and prevent the progression of some major causes of morbidity and mortality. ${ }^{17}$

MetS has a wide clinical spectrum in which NAFLD is a moderate severe state. Obesity, insulin resistance, and type 2 diabetes mellitus (T2DM) are major contributors to the development of NAFLD. There is increasing awareness of potential drug-induced liver injury (DILI) risk factors in NAFLD patients for a growing number of drugs ${ }^{18}$ and DILI may present as lesions that resemble those of NAFLD. ${ }^{19}$ Drug-induced fatty liver is featured with mild to moderate serum enzyme elevations, generally in a hepatocellular pattern, as shown by liver biopsy or ultrasound. The clinical presentations, however, are usually absent or mild and nonspecific. Therefore, the diagnosis of the drug-induced fatty liver is usually made based upon laboratory or imaging test results. Although NAFLD is accepted to be a component of MetS, its presence alone may constitute a cardiovascular risk factor independent from the classic risk factors such as insulin resistance or obesity. ${ }^{20}$

\section{Clinical evidence for aaps-induced mets and NAFLD The prevalence of mets in aaps-treated patients}

A cross-sectional study in Malaysia revealed that patients with schizophrenia receiving antipsychotics have a very high incidence $(46.7 \%)$ of MetS. ${ }^{21}$ But, the prevalence of this syndrome varies in a wide range from $14.7 \%$ to $69.3 \%$ in societies different from European and North American ones (termed non-Euro-American societies [NEAS]). The incidence even reached up to $20 \%$ after 1 year of treatment with an $\mathrm{AAP}^{22}$ The MetS development profile in AAPstreated patients in NEAS seemed to be comparable to that in European and North American societies. ${ }^{23}$ The MetS prevalence in the Italian general population quoted around $26 \%$, relatively low. ${ }^{24}$ A study in Shanghai, China, found that the prevalence of MetS in a total of 468 schizophrenia patients taking clozapine was about $43 \%$, with $40.3 \%$ in males and $51.2 \%$ in females. ${ }^{25}$ Although it varies considerably in the studies cited above, the overall prevalence of AAPs-induced MetS is high. Therefore, the AAPs-induced 
MetS is thought to be a risk factor associated with the development of CVD and excess mortality as mentioned above. $^{14-16}$

\section{Weight gain}

Weight gain has been accepted as common side effect of antipsychotic therapy, reported in up to $50 \%$ of patients receiving long-term treatment for schizophrenia. ${ }^{26}$ Early in 1992, a clinical observation with clozapine treatment for 16 weeks reported that $38 \%$ of the patients experienced marked weight gains and $29 \%$ had moderate weight gains. The improvements in BPRS (brief psychiatric rating scale) total score and composite negative symptom score were significantly greater for the eight patients with marked weight gains than for the other 13 patients. $^{27}$ Following this study, many researchers investigated the AAPs-induced weight gain and MetS. In 1997, a case study reported obesity, liver enzyme abnormalities, and confirmatory evidence of fatty liver in 2 of 13 psychotic children treated with risperidone. ${ }^{28}$ An association between long-term risperidone therapy and the development of obesity, liver dysfunction, and steatohepatitis were observed in these two patients. In 2002, a study of 25 children with pervasive developmental disorder reported that olanzapine $(10.7 \mathrm{mg} /$ day) induced an average weight gain of $4.7 \mathrm{~kg}$ after 12 weeks of treatment, increasing about $10 \%$ of the body weight. ${ }^{29}$ A similar open-label study with risperidone in autistic children showed an average weight gain of $3.2 \mathrm{~kg}$ after the treatment for 4 weeks. ${ }^{30}$ However, the weight gain in a sample of elderly patients treated with olanzapine was insignificant, ${ }^{31}$ although this study was retrospective and limited by a small sample size. In Alzheimer's disease patients with psychosis, olanzapine treatment led to a modest weight gain $(0.8 \mathrm{~kg})$ over 6 weeks, whereas placebo-treated patients in the study showed a weight loss of $0.19 \mathrm{~kg}^{32}$

Of the AAPs, olanzapine showed higher rates of clinically significant ( $>7 \%$ of body weight) weight gain compared with the others. In contrast, ziprasidone had the least impact on weight. Clozapine was more effective than risperidone, but comparable to olanzapine, in causing weight gain. Immediate-release quetiapine was comparable to risperidone but caused greater weight gain than ziprasidone. No significant difference was found between ziprasidone and risperidone. Data for aripiprazole were limited and no comparative evidence for paliperidone was found. ${ }^{4}$

\section{Hyperglycemia and T2DM}

Long before the introduction of antipsychotic drugs, researchers reported an increased prevalence of hyperglycemia and/or
T2DM in schizophrenic patients. ${ }^{33}$ Then, an increase of diabetes prevalence was reported in patients treated with antipsychotics after the introduction of the first neuroleptics in the 1950s, and the same alarms occurred in the 1990s when AAPs were applied in clinical practice. ${ }^{34}$ Persistent reports have linked AAPs with impaired glucose metabolism, exacerbation of existing type 1 and 2 diabetes, new-onset T2DM, and diabetic ketoacidosis. The strength of the association between antipsychotics and diabetes varies across individual medications, with the largest number of reports for chlorpromazine, clozapine, and olanzapine. ${ }^{35}$ Recent controlled studies suggest that antipsychotics can impair glucose regulation by decreasing insulin action. Although the AAPs-induced weight gain may help to explain some treatment-related changes in glucose metabolism, case reports and controlled studies suggest that clozapine and olanzapine treatment may also be associated with adverse effects on glucose metabolism independent of adiposity. One example of case reports described a 31-year-old patient with schizophrenia who developed severe hyperglycemia and ketosis 3 months after starting clozapine therapy. After resolution of his ketoacidosis, clozapine was discontinued. Two months later, his glycosylated hemoglobin (HbAlc) level normalized. Clozapine was restarted, and $72 \mathrm{hrs}$ later, the patient's fasting glucose level increased again. Discontinuation of clozapine led again to normalization of the patient's glucose level. ${ }^{36}$ In a controlled study, olanzapinetreated patients had significant (1.0-1.5 SDs) glucose elevations relative to the patients who received TAPs, or the untreated healthy control subjects. Clozapine-treated patients showed glucose elevations at fasting and 75 mins after load, as compared to the patients received TAPs, or untreated control subjects. ${ }^{37}$ Similarly, the proportion of patients with elevated blood glucose among the schizophrenia patients treated with antipsychotics was significantly higher than that of diabetics in the corresponding age groups in the general population. The comparison made in different groups of antipsychotics revealed that those treated with TAPs had a lower risk of developing diabetes mellitus than those who received dibenzodiazepine derivates like clozapine and olanzapine. ${ }^{38}$ In a recent meta-analysis with thirteen studies including 185,105 youth exposed to antipsychotics, it was concluded that antipsychotic exposure significantly increased the incidence rate ratio of $\mathrm{T} 2 \mathrm{DM}$ relative to healthy controls and psychiatric controls. Olanzapine treatment and antipsychotic exposure time were the main modifiable risk factors for T2DM development in antipsychotic-exposed youth. The authors suggested that antipsychotics should be judiciously used and their efficacy and safety should be monitored proactively. ${ }^{39}$ 


\section{Dyslipidemia}

Lipid metabolism is an importance of MetS. Previous studies have consistently showed marked elevations of serum triglyceride levels with modest alterations in other lipid profiles associated with AAPs administration. ${ }^{40-42}$ In addition, as mentioned above, individual AAPs differ in their liability to induce hypertriglyceridemia. ${ }^{43-45}$ Generally, clozapine and olanzapine have the highest propensity to increase triglyceride levels; quetiapine and risperidone are associated with moderate risk for hyperlipidemia; ziprasidone and aripiprazole have minimal risk.

AAPs-induced elevation in lipid levels has significant health consequences as it increases risk for MetS, CVD, obesity, NAFLD, and acute pancreatitis. In respect to the latter case, several cases of olanzapine associated pancreatitis have been reported since $2000,{ }^{46-50}$ including two reports of acute necrotizing pancreatitis. ${ }^{47,49}$ In the case reported by Rizos et al, ${ }^{49}$ the patient was a 22 -year-old man treated with olanzapine for 6 months at a dose regimen of $10 \mathrm{mg}$ /day because of schizophrenia. He had no preexisting predisposing factors for pancreatitis but showed the $759 \mathrm{C} / \mathrm{T}$ polymorphism of HTR2C gene detected shortly after his discharge from the hospital. This polymorphism was hypothesized to increase the susceptibility of the patient to extreme triglyceride elevation following olanzapine treatment and consequent pancreatic injury.

\section{Nafld}

Although NAFLD is accepted to be a component of MetS, its presence alone constitutes a cardiovascular risk factor independent from the classic risk factors such as insulin resistance or obesity. ${ }^{51}$ All these components of MetS have been associated with AAPs use in patients with schizophrenia as reviewed above. However, few clinical researches were reported on the association of NAFLD and AAPs use in patients with schizophrenia. Of the recently published studies, a case-control research demonstrated that patients with schizophrenia were 4.42 times more likely to have liver disease. ${ }^{52}$ A large-scale retrospective study reported a higher prevalence of liver disease and of alcohol-related cirrhosis in veterans with schizophrenia. ${ }^{53}$ Furthermore, a community-based study in patients with serious mental illness reported an OR of 2.69 in comparing schizophrenia patients to a reference group with liver problems. ${ }^{54}$ Similarly, Hsu et al reported that the prevalence and incidence of chronic liver disease in schizophrenia patients were 1.27 and 1.15 times as high as those of the general population, respectively. ${ }^{55}$ In a recent clinical trial with a large epidemiological cohort of drug-naïve patients with first episode nonaffective psychosis, the potential development of NAFLD during the first 3 years of antipsychotic treatment was analyzed. At baseline, none of the patients showed significant liver fibrosis. At 3 years follow-up, however, 25.1\% individuals showed a FLI (fatty liver index) score $\geq 60$, which is a predictor of steatosis. Although none of the patients included in the analysis displayed significant liver fibrosis, NAFLD was an early indicator of liver steatosis in a considerable proportion of patients during the follow-up period. ${ }^{56}$ Given the link between cardiometabolic risk, NAFLD, and antipsychotic medication, early detection of potential NAFLD development using non-invasive techniques could be worth considering in clinical practice. These patients should be monitored to treat liver disease and the potential underlying cardiovascular disease risk factors. ${ }^{57}$

\section{Efficacy and safety profile of sertindole}

Sertindole is a novel AAP. It was developed in the 1990s and first authorized in the United Kingdom in 1996. But, it was suspended by Lundbeck in November $1998{ }^{58}$ because of Q-T prolongation reported in three studies. ${ }^{59} \mathrm{Q}-\mathrm{T}$ prolongation is a risk factor for torsade de pointes, an arrhythmia that may progress to ventricular fibrillation. ${ }^{60}$ Although sertindole is not the only antipsychotic that may lead to Q-T prolongation, the use of it should be contraindicated in patients suffering from underlying cardiac diseases or hypokalemia and in those patients undergoing concomitant treatment with other medication known to prolong the Q-T interval.

After extended re-evaluation of its safety and risk-benefit profile, sertindole was reintroduced in the market in 2006. Comparative studies have shown sertindole to be an efficacious AAP, ${ }^{61,62}$ associated with placebo-level EPS. ${ }^{63}$ Moreover, sertindole exerts a beneficial effect on the cognitive disturbances associated with schizophrenia. ${ }^{64-66}$ In a review article by Karamatskos et al, ${ }^{67}$ sertindole was shown to be comparable to haloperidol and risperidone on positive symptoms, but superior on negative symptoms. It is generally well tolerated but is associated with a dose-related QTc interval prolongation.

The data in the literature on possible metabolic side effects of sertindole are scanty. In the sertindole cohort prospective study the short- and long-term metabolic safety of sertindole was compared to that of risperidone 
in a subset of patients. From baseline to last assessment (up to 60 weeks), comparable weight gains (1.8 and $1.7 \mathrm{~kg}$ for sertindole and risperidone, respectively) were observed. The both treatments did not result in clinic relevant changes in triglycerides, total cholesterol, HDLcholesterol, LDL-cholesterol, plasma glucose and blood pressure. No patient in either of the groups developed type 2 diabetes during the study. ${ }^{68}$ In an open-label, noncomparative, flexible-dose study carried out in 11 European countries, the safety and tolerability of sertindole in the long-term treatment of schizophrenia were assessed. The most common adverse events were fatigue and weight gain, both with incidences of $14 \%$.

\section{Metabolic effects and NAFLD of aaps in animal studies}

So far, preclinical research has extensively addressed metabolic effects of AAPs. In rodent experiments, metabolic abnormalities induced by antipsychotics, specifically weight gain, glucose metabolism dysregulation and altered lipid profile have been described. ${ }^{69}$ Here we focused on some individual risk factors for AAP-induced metabolic side effects, including weight gain, glucose dysregulation, lipid disturbances, and NAFLD.

\section{Weight gain}

Earlier animal studies of antipsychotics-induced obesity mainly involved sub-chronic or chronic administration of sulpiride, which is a specific blocker of D2-D3 receptors. In 2002, Baptista et $\mathrm{al}^{70}$ reported that acute administration (12 days) of risperidone induced significant bodyweight gain only in female rats. Similarly, olanzapine administration, even a single day of treatment, promoted bodyweight gain in female rats and the weight gain was rapidly reversible. ${ }^{71}$ A subsequent study of the same group suggested that olanzapine-induced hyperphagia acts as an initial stimulus leading to weight gain, visceral adiposity and subsequent insulin resistance. ${ }^{72}$ Other authors have also shown consistently that olanzapine induces weight gain in female rats and mice. Such sex-dependent effects questioned whether rodent models mimic the clinical situation as there is no evidence that antipsychotics-induced weight gain in the clinic is only seen in females. ${ }^{73}$ This issue is highlighted further by the finding that administration of clozapine over a very wide dose range failed to induce weight gain, which is in contrast to the clinical finding that clozapine induces substantial weight gain in humans. ${ }^{74}$

\section{Glucose dysregulation}

Glucose dysregulation is characterized by higher levels of fasting blood glucose and glucose intolerance. In vivo models commonly reported higher levels of fasting plasma glucose in rats after either acute or chronic treatment with most AAPs. ${ }^{69,75,76}$ Elevated and sustained plasma glucose levels could be attributable either to decreased insulinmediated glucose uptake or to increased endogenous hepatic glucose production/output (HGO). Indeed, quetiapine and clozapine were shown to induce derangements in glucose homeostasis by acutely promoting glucagon secretion and HGO in the rat. ${ }^{77}$ The two drugs also acutely reduced glucagon-like peptide- ${ }^{1}$ production, but increased glucagon release in obese rats. ${ }^{78}$ These results confirmed that the liver is the primary site of increased glucose output as shown in a previous study. ${ }^{79}$ Also, they are consistent with the finding in a study that olanzapine acutely induces HGO. ${ }^{80}$ In a follow-up study, the same group stated that AAPs differ in their propensities to impair glucose production; of them clozapine and olanzapine increased $\mathrm{HGO}$, whereas ziprasidone decreased HGO. ${ }^{81}$ In another study, AAPs produced significant dose and time dependent effects on fasting plasma glucose, insulin concentrations, and glucose intolerance. The greatest effect on glucose dysregulation was noted primarily with clozapine and olanzapine. ${ }^{69}$

\section{Adiposity and dyslipidemia}

Increased adiposity results from enhanced lipid storage and/or impaired lipid mobilization. In support of this concept, rat adipocytes in cultures treated with olanzapine and clozapine showed enhanced lipogenesis. ${ }^{82,83}$ Similarly, adipocytes isolated from chronic olanzapine-treated rats presented an increase in fatty acid synthase expression. ${ }^{84}$ Moreover, the lipolytic capacity of the adipose tissue was impaired in response to AAPs, as demonstrated by reduced expression of hormone-sensitive lipase. In cultured human hepatocytes and in rat liver, acute exposure to clozapine resulted in lipogenesis stimulation through activation of SREBPs (sterol regulatory element-binding proteins). ${ }^{85,86}$ In addition, adipocytes from olanzapine-treated rats tended to be larger relative to those in untreated rats. This could result in the release of adipocytes content, eg triacylglycerides, into the circulation thereby increasing the risk of dyslipidemia. ${ }^{87}$ Furthermore, AAPs exerted direct effects on mature rat adipocytes, caused an increase in lipogenesis (ie triacylglyceride accumulation). ${ }^{83,84}$ In other studies, 
clozapine and olanzapine enhanced lipogenesis in murine preadipocytes $^{83,88}$ and adipocytes, particularly in those located in white visceral adipose tissue. ${ }^{84,86}$

Besides increasing lipogenesis and decreasing lipolysis, AAPs were shown to stimulate the differentiation of preadipocytes into adipocytes, thus increasing adipose tissue mass. ${ }^{89,90}$ For example, olanzapine and clozapine upregulated the expression of transcription factors, such as peroxisome proliferator-activated receptor- $\gamma$ (PPAR- $\gamma$ ), CCAAT-enhancer binding protein $\beta(\mathrm{C} / \mathrm{EBP} \beta)$ in human and rodent adipocytes, which are essential for adipocyte differentiation. Moreover, the two drugs elevated levels of the adipocyte determination and differentiation factor 1/SREBP1C (ADD1/SREBP-1C). ${ }^{85,86,91}$ Last, but not least, AAPs increase mitochondrial ROS production ${ }^{92}$ and induce oxidative stress in cell lines and tissues, ${ }^{93-95}$ thus influencing the balance between proliferation and differentiation of adipocyte progenitor cells.

\section{Nafld}

Liver and adipose tissue play important roles in energy and lipid metabolism. As such, it was proposed that antipsychotic-mediated lipogenic effects in peripheral tissues are relevant for the risk of metabolic adverse effects associated with some of these drugs. ${ }^{85,86}$ In line with this suggestion, AAPs increased the synthesis of phospholipid, triacylglycerides and free fatty acids in primary mouse and rat hepatocytes ${ }^{96,97}$ and human hepatocyte cell lines. ${ }^{98}$ Moreover, acute exposure of rats to clozapine increased the hepatic content of phospholipids and triglycerides. ${ }^{85}$ These complex lipids are stored in lipid droplets and membrane structures in hepatocytes and may be secreted into the circulation as very low-density lipoprotein particles. Indeed, olanzapine- and aripiprazole-treated rats showed significantly increased fatty infiltration of liver (steatosis) compared with the control group, although the aripiprazole-treated group showed less steatosis compared with olanzapine. The mean non-alcoholic steatohepatitis score and fibrosis of the olanzapine group were significantly increased compared to the aripiprazole group. In addition, liver from the olanzapine group showed large fat droplets in perinuclear region, between cisternae of the rough endoplasmic reticulum, and in the space of Disse. ${ }^{99}$ In a more recent study, olanzapine-administered (for 2 weeks) rats showed severe liver damage indicated by loss of normal architecture, vacuolar degeneration of hepatocytes and fatty changes in the liver. ${ }^{100}$ In a study from our group, daily intraperitoneal injection of clozapine for 6 weeks significantly increased plasma levels of triglycerides, led to hepatic steatosis and fibrosis along with increased activities of alanine aminotransferase and aspartate aminotransferase in male Wistar rats, but showed no effects on blood glucose, insulin levels, and total cholesterol. $^{101}$

\section{Mechanisms involved in the AAPs- induced mets and NAFLD Weight gain and obesity}

Although the molecular mechanisms for AAPs-induced weight gain and obesity remain unclear, the antipsychotics-induced weight gain has been believed to result from the induction or aggravation of an imbalance between energy intake (ingested calories) and energy expenditure (exercise). One of the proposed mechanisms points to the ability of AAPs to induce hyperphagia by acting as moderate antagonists of monoamine neurotransmitter receptors, including those of histamine, serotonin, or dopamine, in the central nervous system. ${ }^{102}$

It is known that appetite and food intake are regulated by the action of hypothalamic monoaminergic neurotransmitters and neuropeptides. Particularly, neuropeptide $\mathrm{Y}$ (NPY) and $\alpha$-melanocyte-stimulating hormone are orexigenic peptides, whereas serotonin (5-HT) enhances satiety and regulates food intake, and the antagonism of the 5HT2C receptor prevents satiety. ${ }^{103}$ In addition, histamine H1 receptor blockade increases appetite; this receptor seems to be required for the action of leptin, a peptide hormone secreted by adipocyte cells. This peptide plays a critical role in regulating adipose tissue mass and energy balance. ${ }^{104,105}$ The actions of other hormones in addition to leptin may also be affected, and perhaps disrupted, by antipsychotic drug treatment.

Adiponectin is an orexigenic hormone secreted by adipose tissue, with opposite effects to that of leptin on feeding behavior. Its expression can be influenced by adiposity, insulin resistance and T2DM. ${ }^{106,107}$ In schizophrenia patients treated with AAPs, lower adiponectin level was associated with insulin resistance, increased inflammation as determined by C-reactive protein, and elevated serum leptin. ${ }^{108}$ In a recent meta-analysis by Bartoli et al, ${ }^{109}$ olanzapine or clozapine treatment was associated with decreases in adiponectin. Ghrelin is another hormone secreted by the stomach. Like adiponectin, ghrelin opposes the effects of leptin in the hypothalamus. In a recent study, olanzapine was shown to promote 
appetite by enhancing ghrelin-receptor signaling, ${ }^{110}$ long term olanzapine administration inhibited post-prandial reduction of ghrelin in rats. ${ }^{11}$

In some cases, adiposity may develop without hyperphagia. ${ }^{112}$ Therefore, it is plausible that the other side effects, such as sedation and muscle stiffness, decreased activity (exercise) and energy expenditure, may be involved in the AAPs-induced weight gain. ${ }^{102}$ Moreover, AAPs may exert direct effects on mature rat adipocytes thus causing an increase in lipogenesis, ie triacylglyceride accumulation. ${ }^{83,84}$ For example, clozapine and olanzapine enhanced lipogenesis in murine preadipocytes ${ }^{85,87}$ and adipocytes, ${ }^{84,85}$ particularly in those located in white visceral adipose tissue.

It is noteworthy that obesity, particularly visceral fat, is associated with a low-grade inflammatory state. ${ }^{113}$ These low-grade inflammatory alterations have been associated with an increased abundance of macrophages in adipose tissue in humans and rodents, ${ }^{113}$ which may contribute to the production of inflammatory molecules and induce the development of obesity-related comorbidities. ${ }^{114,115}$ Although the mechanism that underlies obesity in schizophrenia patients is not clear, antipsychotic medications, chronic low-grade inflammation, lifestyle, and genetic vulnerability are suggested as risk factors for overweight and obesity. ${ }^{116,117}$ In line with this suggestion, pro-inflammatory cytokine levels were raised in female schizophrenia patients treated with clozapine, ${ }^{118}$ whereas aripiprazole limited inflammatory processes while reduced the risk of metabolic abnormalities via enhancing anti-inflammatory signaling $^{119}$ In a most recent study, however, olanzapine increased levels of IL-6 and IL-27 during the first episode of schizophrenia while improved psychological symptoms and particularly the negative symptoms in the patients. ${ }^{120}$ Therefore, antipsychotics may exert anti-inflammatory and/or pro-inflammatory effects, which may explain response variability and the variable emergence of adverse effects, such as MetS. ${ }^{121}$

\section{Glucose dysregulation}

Early in 1972, Baker and Rogers demonstrated that chlorpromazine was able to block glucose uptake into human erythrocytes. $^{122}$ Following that, several studies showed that acute exposure to AAPs (clozapine and risperidone) inhibited GLUT1- and GLUT3-mediated glucose uptake in PC12 cells. ${ }^{123-126}$ Clozapine and fluphenazine inhibited GLUT4-mediated glucose transport in the rat muscle L6 cell line. ${ }^{123}$ Later, clozapine was shown to reduce insulinstimulated glucose transport into rat adipocytes. ${ }^{75}$
Similarly, clozapine and olanzapine decreased insulin-stimulated glucose transport into rat pre-adipocytes and primary adipocytes. ${ }^{84}$ In line with the above studies, antipsychotics in vitro effectiveness in inhibiting glucose transport was correlated to their in vivo effectiveness in raising plasma glucose in mice injected with an intraperitoneal bolus of these drugs. ${ }^{126}$ In addition, a decrease in GLUT4 mRNA expression and recruitment to cell surface may contribute to the inhibition of glucose transport in adipose tissue induced by antipsychotics treatment. ${ }^{127}$

Protein kinase B (Akt) may be rapidly activated by insulin and able to mediate the translocation of GLUT4 onto the plasma membrane. ${ }^{128}$ Interestingly, activation of $\mathrm{D} 2$ receptor family was shown to stimulate the assembly of a complex containing $\beta$-arrestin 2 , protein phosphatase $2 \mathrm{~A}$ and Akt. ${ }^{129,130}$ In addition, mitochondrial dysfunction may play a key role in the development of insulin resistance and diabetes associated to the use of antipsychotics, ${ }^{131,132}$ given the finding that antipsychotics negatively affected mitochondrial function, altered the function of key metabolic enzymes and negatively affected carbon metabolism and/or electron transport during oxidative phosphorylation, thereby decreasing glucose metabolism. ${ }^{133-135}$

\section{Dyslipidemia and NAFLD}

AAPs disturb lipid metabolism through their effects on SREBPs. SREBPs exist as two homologous proteins: SREBP-1 and SREBP-2. SREBP-1 is known as the key regulator of fatty acid synthesis and a molecular link between lipid metabolism, insulin action and obesity at the gene regulatory level; ${ }^{136}$ whereas SREBP-2 is the main regulator of cholesterol metabolism. ${ }^{137}$ It was reported that olanzapine, clozapine and risperidone elicited significant upregulation of SREBP-1, SREBP-2, and their downstream target genes thus led to increased lipid and cholesterol synthesis in primary rat hepatocytes and mouse liver. ${ }^{95,97,138}$ Even a single intraperitoneal injection of clozapine or olanzapine induced elevation of serum free fatty acids, followed by hepatic accumulation of lipids. ${ }^{85,127}$ In a recent study, clozapine and risperidone significantly increased hepatic expressions of SCAP/SREBP and the parallel inhibition of PGRMC1/INSIG-2, while markedly elevated levels of serum lipid and hormone parameters in rats. ${ }^{139}$

Another potential mechanism contributing to the increased risk for AAPs-induced hyperlipidemia is a deficiency in long-chain omega-3 (LC n-3) fatty acids, including eicosapentaenoic acid (EPA) and docosahexaenoic acid (DHA). Specifically, increasing LC n-3 fatty acid 
status through dietary supplementation is efficacious in reducing elevated triglycerides levels. ${ }^{140}$ Indeed, LC n-3 fatty acids have been approved by the United States FDA for the treatment of hypertriglyceridemia. Preclinical studies have reported that low LC n-3 fatty acid status is associated with greater liver triglycerides content and secretion in different rodent models. ${ }^{141-144}$ Clinical studies have shown that patients with obesity and NAFLD exhibit significantly lower LC n-3 fatty acid levels in liver and red blood cells. ${ }^{145,146}$ These results are in line with the findings in schizophrenia patients who received AAPs and showed significantly lower LC n-3 fatty acid levels compared with healthy controls. ${ }^{147-149}$ Last, but not least, increasing LC n-3 fatty acid status through dietary supplementation mitigated the elevation in serum triglycerides in AAPs-treated patients. ${ }^{150,151}$ Together, these data suggest that low LC n-3 fatty acid status may represent a modifiable risk factor for AAPs-induced elevations in triglycerides production, weight gain and NAFLD. In support of this suggestion, low LC n-3 fatty acid status exacerbated risperidone-induced hepatic steatosis by augmenting the expression and activity of stearoyl-CoA desaturase-1, which plays a pivotal role in triglycerides biosynthesis. ${ }^{152}$

\section{Concluding remarks}

The AAPs represent an effective therapeutic option for a wide range of psychotic disorders, especially the schizophrenia. Their growing use has raised questions about their tolerability over the metabolic disturbance and even NAFLD. Amounts of clinical research have put forward tendency to obesity, glycometabolic disturbance, dyslipidemia and fatty liver in AAPs treatment. Experimental studies reveal that 5-HTR, H1R, GLUT play important roles in the process circuitously, the SREBP can regulate lipid biosynthesis directly to induce NAFLD, and LC n-3 fatty acid level is positively correlated with these effects. Many other factors should be concerned in this process as well.

Unlike weight gain and insulin resistance, dyslipidemia and NAFLD can easily be ignored because of their diagnostic difficulty, mild index abnormality and dormant clinical symptoms. However, the presence of steatosis and ballooning degeneration, inflammation and variable degrees of fibrosis is generally referred to as non-alcoholic steatohepatitis and is considered a more severe part of the spectrum of NAFLD, being potentially progressive and leading to advanced fibrosis or cirrhosis. What is worse, in clinical practice most patients starting AAPs do not receive recommended regular glucose or lipid screening or monitoring. At present, not only the symptom improvement, but also the overall quality of patients' lives should get more attention.

Some opinions are put forward here. First, monitoring weight gain is important but insufficient. Periodic monitoring of blood sugar may also be required during antipsychotic therapy, particularly for drugs with high diabetic liabilities such as olanzapine and clozapine. Monitoring of alanine aminotransferase levels and regular ultrasound, computerized tomography or magnetic resonance imaging tests are recommended, particularly in at-risk individuals. Therefore, careful assessment of a patient's clinical profile is warranted, in addition to routine monitoring of liver tests, to identify atrisk individuals (underlying liver disease, alcohol use, polypharmacy, drug-drug interactions, high daily AAPs dose, and metabolic abnormalities). Healthcare practitioners should educate patients to recognize manifestations of liver injury. So far, the life style modifications, nutritional consulting (specially, high LC n-3 fatty acid diet and regularly vitamin D supplement are recommended), and polypharmacological interventions have proved certain efficacy against AAPs-induced metabolic side effects.

\section{Disclosure}

Dr. $\mathrm{Xu}$ received grants from Natural Science Foundation of Guangdong Province (2016A030313067) and $\mathrm{Li}$ Kashing Foundation (43209502). The authors report no other conflicts of interest in this work.

\section{References}

1. Turner T. Chlorpromazine: unlocking psychosis. BMJ. 2007;334(Suppl 1):S7. doi:10.1136/bmj.39034.609074.94

2. Maric NP, Jovicic MJ, Mihaljevic M, Miljevic C. Improving current treatments for schizophrenia. Drug Dev Res. 2016;77:357-367. doi: $10.1002 /$ ddr. 21337

3. Warnez S, Alessi-Severini S. Clozapine: a review of clinical practice guidelines and prescribing trends. BMC Psychiatry. 2014;14:102. doi:10.1186/1471-244X-14-102

4. McDonagh M, Peterson K, Carson S, Fu R, Thakurta S. Drug Class Review. A typical antipsychotic drugs: final update 3 report. Drug Class Rev. 2010.

5. Hermann RC, Yang D, Ettner SL, Marcus SC, Yoon C, Abraham M. Prescription of antipsychotic drugs by office-based physicians in the United States, 1989-1997. Psychiatr Serv. 2002;53:425-430. doi:10.1176/ appi.ps.53.4.425

6. Carbon M, Hsieh C-H, Kane JM, Correll CU. Tardive dyskinesia prevalence in the period of second-generation antipsychotic use: a meta-analysis. J Clin Psychiatry. 2017;78:e264-e278. doi:10.4088/ JCP.16r10832

7. Zhang JP, Gallego JA, Robinson DG, Malhotra AK, Kane JM, Correll $\mathrm{CU}$. Efficacy and safety of individual second-generation vs. first-generation antipsychotics in first-episode psychosis: a systematic review and meta-analysis. Int $J$ Neuropsychopharmacol. 2013;16:1205-1218. doi:10.1017/S1461145712001277 
8. Mitchell AJ, Vancampfort D, Sweers K, van Winkel R, Yu W, De Hert M. Prevalence of metabolic syndrome and metabolic abnormalities in schizophrenia and related disorders-a systematic review and meta-analysis. Schizophr Bull. 2013;39:306-318. doi:10.1093/ schbul/sbr148

9. De Hert M, Yu W, Detraux J, Sweers K, van Winkel R, Correll CU. Body weight and metabolic adverse effects of asenapine, iloperidone, lurasidone and paliperidone in the treatment of schizophrenia and bipolar disorder: a systematic review and exploratory metaanalysis. CNS Drugs. 2012;26:733-759. doi:10.2165/11634500000000000-00000

10. Hasnain M, Fredrickson SK, Vieweg WV, Pandurangi AK. Metabolic syndrome associated with schizophrenia and atypical antipsychotics. Curr Diab Rep. 2010;10:209-216. doi:10.1007/s11892-010-0112-8

11. Newcomer JW. Second-generation (atypical) antipsychotics and metabolic effects: a comprehensive literature review. CNS Drugs. 2005;19(Suppl 1):1-93. doi:10.2165/00023210-200519001-00001

12. Newcomer JW. Antipsychotic medications: metabolic and cardiovascular risk. J Clin Psychiatry. 2007;68(Suppl 4):8-13.

13. Rummel-Kluge C, Komossa K, Schwarz S, et al. Head-to-head comparisons of metabolic side effects of second generation antipsychotics in the treatment of schizophrenia: a systematic review and meta-analysis. Schizophr Res. 2010;123:225-233. doi:10.1016/ j.schres.2010.07.012

14. Galassi A, Reynolds K, He J. Metabolic syndrome and risk of cardiovascular disease: a meta-analysis. $\mathrm{Am} J \mathrm{Med}$ 2006;119:812-819. doi:10.1016/j.amjmed.2006.02.031

15. Gami AS, Witt BJ, Howard DE, et al. Metabolic syndrome and risk of incident cardiovascular events and death: a systematic review and meta-analysis of longitudinal studies. $\mathrm{J}$ Am Coll Cardiol. 2007;49:403-414. doi:10.1016/j.jacc.2006.09.032

16. Mottillo S, Filion KB, Genest J, et al. The metabolic syndrome and cardiovascular risk a systematic review and meta-analysis. $J$ Am Coll Cardiol. 2010;56:1113-1132. doi:10.1016/j.jacc.2010.05.034

17. Eckel RH, Alberti KG, Grundy SM, Zimmet PZ. The metabolic syndrome. Lancet. 2010;375:181-183. doi:10.1016/S0140-6736 (09)61794-3

18. Massart J, Begriche K, Moreau C, Fromenty B. Role of nonalcoholic fatty liver disease as risk factor for drug-induced hepatotoxicity. J Clin Transl Res. 2017;3:212-232. doi:10.18053/jctres.03.2017S1.006

19. Tarantino G, Conca P, Basile V, et al. A prospective study of acute drug-induced liver injury in patients suffering from non-alcoholic fatty liver disease. Hepatol Res. 2007;37:410-415. doi:10.1111/ j.1872-034X.2007.00072.x

20. Slim M, Medina-Caliz I, Gonzalez-Jimenez A, et al. Hepatic safety of atypical antipsychotics: current evidence and future directions. Drug Saf. 2016;39:925-943. doi:10.1007/s40264-016-0436-7

21. Said MA, Sulaiman AH, Habil MH, et al. Metabolic syndrome and cardiovascular risk among patients with schizophrenia receiving antipsychotics in Malaysia. Singapore Med J. 2012;53:801-807.

22. Bou KR. Atypical antipsychotic drugs, schizophrenia, and metabolic syndrome in non-Euro- American societies. Clin Neuropharmacol. 2012;35:141-147. doi:10.1097/WNF.0b013e31824d5288

23. McEvoy JP, Meyer JM, Goff DC, et al. Prevalence of the metabolic syndrome in patients with schizophrenia: baseline results from the Clinical Antipsychotic Trials of Intervention Effectiveness (CATIE) schizophrenia trial and comparison with national estimates from NHANES III. Schizophr Res. 2005;80:19-32. doi:10.1016/j. schres.2005.07.014

24. Vanuzzo D, Lo NC, Pilotto L, et al. Cardiovascular epidemiologic observatory 2008-2011: preliminary results. G Ital Cardiol (Rome). 2010;11:25S-30S.

25. Zhang Y, Chen M, Chen J, et al. Metabolic syndrome in patients taking clozapine: prevalence and influence of catechol-O-methyltransferase genotype. Psycho- Pharmacology (berl). 2014;231:2211-2218. doi:10.1007/s00213-013-3410-4
26. Baptista T. Body weight gain induced by antipsychotic drugs: mechanisms and management. Acta Psychiatr Scand. 1999;100:3-16.

27. Leadbetter R, Shutty M, Pavalonis D, Vieweg V, Higgins P, Downs M. Clozapine-induced weight gain: prevalence and clinical relevance. $\mathrm{Am}$ J Psychiatry. 1992;149:68-72. doi:10.1176/ajp.149.1.68

28. Kumra S, Herion D, Jacobsen LK, Briguglia C, Grothe D. Case study: risperidone-induced hepatotoxicity in pediatric patients. $J$ Am Acad Child Adolesc Psychiatry. 1997;36:701-705. doi:10.1097/00004583-199705000-00022

29. Kemner C, Willemsen-Swinkels SH, de Jonge M, Tuynman-Qua H, van Engeland H. Open-label study of olanzapine in children with pervasive developmental disorder. $J$ Clin Psychopharmacol. 2002;22:455-460.

30. Gagliano A, Germanò E, Pustorino G, et al. Risperidone treatment of children with autistic disorder: effectiveness, tolerability, and pharmacokinetic implications. J Child Adolesc Psychopharmacol. 2004;14:39-47. doi:10.1089/104454604773840472

31. Madhusoodanan S, Suresh P, Brenner R, Pillai R. Experience with the atypical antipsychotics risperidone and olanzapine in the elderly. Ann Clin Psychiatry. 1999;11:113-118.

32. Street JS, Clark WS, Gannon KS, et al. Olanzapine treatment of psychotic and behavioral symptoms in patients with Alzheimer disease in nursing care facilities: a double-blind, randomized, placebo-controlled trial. The HGEU study group. Arch Gen Psychiatry. 2000;57:968-976.

33. Barahal HS, Freeman N. Sudden graying of hair, alopecia, and diabetes mellitus of psychogenic origin. Psychiatr Q. 1946;20:31-38.

34. Gury C. Schizophrenia, diabetes mellitus and antipsychotics. Encephale. 2004;30:382-391.

35. Haupt DW, Newcomer JW. Hyperglycemia and antipsychotic medications. J Clin Psychiatry. 2001;62(Suppl 27):15-26; discussion 40-41.

36. Colli A, Cocciolo M, Francobandiera F, Rogantin F, Cattalini N. Diabetic ketoacidosis associated with clozapine treatment. Diabetes Care. 1999;22:176-177. doi:10.2337/diacare.22.1.176a

37. Newcomer JW, Haupt DW, Fucetola R, et al. Abnormalities in glucose regulation during antipsychotic treatment of schizophrenia. Arch Gen Psychiatry. 2002;59:337-345.

38. Wetterling T, Schneider B, Weber B. Dyslipidemia and schizophrenia. Psychiatr Prax. 2007;34:223-229. doi:10.1055/s-2006-940056

39. Galling B, Roldán A, Nielsen RE, et al. Type 2 diabetes mellitus in youth exposed to antipsychotics: a systematic review and metaanalysis. JAMA Psychiatry. 2016;73:247-259. doi:10.1001/ jamapsychiatry.2015.2923

40. Atmaca M, Kuloglu M, Tezcan E, Ustundag B. Serum leptin and triglyceride levels in patients on treatment with atypical antipsychotics. J Clin Psychiatry. 2003;64:598-604.

41. Meyer JM, Koro CE. The effects of antipsychotic therapy on serum lipids: a comprehensive review. Schizophr Res. 2004;70:1-17. doi:10.1016/j.schres.2004.01.014

42. Wirshing DA, Boyd JA, Meng LR, Ballon JS, Marder SR, Wirshing WC. The effects of novel antipsychotics on glucose and lipid levels. J Clin Psychiatry. 2002;63:856-865.

43. Meyer JM. A retrospective comparison of weight, lipid, and glucose changes between risperidone- and olanzapine-treated inpatients: metabolic outcomes after 1 year. J Clin Psychiatry. 2002;63:425-433.

44. Olfson M, Marcus SC, Corey-Lisle P, Tuomari AV, Hines P, L'Italien GJ. Hyperlipidemia following treatment with antipsychotic medications. Am J Psychiatry. 2006;163:1821-1825. doi:10.1176/ajp.2006.163.10.1821

45. Weiden PJ, Daniel DG, Simpson G, Romano SJ. Improvement in indices of health status in outpatients with schizophrenia switched to ziprasidone. $J$ Clin Psychopharmacol. 2003;23:595-600. doi:10.1097/01.jcp.0000095347.32154.08

46. Doucette DE, Grenier JP, Robertson PS. Olanzapine-induced acute pancreatitis. Ann Pharmacother. 2000;34:1128-1131. doi:10.1345/ aph. 19390 
47. Gupta A, Dadheech G, Yadav D, Sharma P, Gautam S. Metabolic issues in schizophrenic patients receiving antipsychotic treatment. Indian J Clin Biochem. 2014;29:196-201. doi:10.1007/s12291013-0415-z

48. Kerr TA, Jonnalagadda S, Prakash C, Azar R. Pancreatitis following olanzapine therapy: a report of three cases. Case Rep Gastroenterol. 2007;1:15-20. doi:10.1159/000104222

49. Rizos E, Tournikioti K, Alevyzakis E, et al. Acute necrotizing pancreatitis following olanzapine treatment and $759 \mathrm{C} / \mathrm{T}$ polymorphism of HTR2C gene: a case report. In Vivo. 2015;29:529531.

50. Rossor AM, Leech N, Neely RD. Olanzapine-induced chylomicronemia presenting as acute pancreatitis. $J$ Clin Psychopharmacol. 2007;27:395-396. doi:10.1097/01.jcp.000026 4988.55603.bb

51. Targher G, Bertolini L, Padovani R, et al. Prevalence of nonalcoholic fatty liver disease and its association with cardiovascular disease in patients with type 1 diabetes. $J$ Hepatol. 2010;53:713718. doi:10.1016/j.jhep.2010.04.030

52. Carney CP, Jones L, Woolson RF. Medical comorbidity in women and men with schizophrenia: a population-based controlled study. $J$ Gen Intern Med. 2006;21:1133-1137. doi:10.1111/j.15251497.2006.00563.x

53. Fuller BE, Rodriguez VL, Linke A, Sikirica M, Dirani R, Hauser P. Prevalence of liver disease in veterans with bipolar disorder or schizophrenia. Gen Hosp Psychiatry. 2011;33:232-237. doi:10.1016/j.genhosppsych.2011.03.006

54. Sokal J, Messias E, Dickerson FB, et al. Comorbidity of medical illnesses among adults with serious mental illness who are receiving community psychiatric services. $J$ Nerv Ment Dis. 2004;192:421-427.

55. Hsu JH, Chien IC, Lin CH, Chou YJ, Chou P. Increased risk of chronic liver disease in patients with schizophrenia: a populationbased cohort study. Psychosomatics. 2014;55:163-171. doi:10.1016/j.psym.2013.06.001

56. Morlán-Coarasa MJ, Arias-Loste MT, de la Foz VO, et al. Incidence of non-alcoholic fatty liver disease and metabolic dysfunction in first episode schizophrenia and related psychotic disorders: a 3-year prospective randomized interventional study. Psychopharmacology (Berl). 2016;233:3947-3952. doi:10.1007/ s00213-016-4422-7

57. Liu H, Lu HY. Nonalcoholic fatty liver disease and cardiovascular disease. World J Gastroenterol. 2014;20:8407-8415. doi:10.3748/ wjg.v20.i26.8407

58. Moore N, Hall G, Sturkenboom M, Mann R, Lagnaoui R, Begaud B. Biases affecting the proportional reporting ratio (PPR) in spontaneous reports pharmacovigilance databases: the example of sertindole. Pharmacoepidemiol Drug Saf. 2003;12:271-281. doi: $10.1002 /$ pds. 848

59. Hale AS. A review of the safety and tolerability of sertindole. Int Clin Psychopharmacol. 1998;13(Suppl 3):S65-S70.

60. Haddad PM, Anderson IM. Antipsychotic-related QTc prolongation, torsade de pointes and sudden death. Drugs. 2002;62:16491671. doi: 10.2165/00003495-200262110-00006

61. Azorin JM, Strub N, Loft H. A double-blind, controlled study of sertindole versus risperidone in the treatment of moderate-to-severe schizophrenia. Int Clin Psychopharmacol. 2006;21:49-56.

62. Daniel DG, Wozniak P, Mack RJ, McCarthy BG. Long-term efficacy and safety comparison of sertindole and haloperidol in the treatment of schizophrenia. The Sertindole Study Group. Psychopharmacol Bull. 1998;34:61-69.

63. Zimbroff DL, Kane JM, Tamminga CA, et al. Controlled, doseresponse study of sertindole and haloperidol in the treatment of schizophrenia. Sertindole study group. Am J Psychiatry. 1997;154:782-791. doi:10.1176/ajp.154.6.782
64. Gallhofer B, Jaanson P, Mittoux A, Tanghøj P, Lis S, Krieger S. Course of recovery of cognitive impairment in patients with schizophrenia: a randomised double-blind study comparing sertindole and haloperidol. Pharmacopsychiatry. 2007;40:275-286. doi:10.1055/s-2007-990291

65. Idris N, Neill J, Grayson B, et al. Sertindole improves sub-chronic PCP-induced reversal learning and episodic memory deficits in rodents: involvement of 5-HT(6) and 5-HT (2A) receptor mechanisms. Psychopharmacology (Berl). 2010;208:23-36. doi:10.1007/ s00213-009-1702-5

66. Rodefer JS, Nguyen TN, Karlsson JJ, Arnt J. Reversal of subchronic PCP-induced deficits in attentional set shifting in rats by sertindole and a 5-HT6 receptor antagonist: comparison among antipsychotics. Neuropsychopharmacology. 2008;33:2657-2666. doi:10.1038/sj.npp.1301654

67. Karamatskos E, Lambert M, Mulert C, Naber D. Drug safety and efficacy evaluation of sertindole for schizophrenia. Expert Opin Drug Saf. 2012;11:1047-1062. doi:10.1517/14740338.2012.726984

68. De Hert M, Mittoux A, He Y, Peuskens J. Metabolic parameters in the short- and long-term treatment of schizophrenia with sertindole or risperidone. Eur Arch Psychiatry Clin Neurosci. 2011;261:231239. doi:10.1007/s00406-010-0142-x

69. Boyda HN, Tse L, Procyshyn RM, Honer WG, Barr AM. Preclinical models of antipsychotic drug-induced metabolic side effects. Trends Pharmacol Sci. 2010;31:484-497. doi:10.1016/j.tips.2010.07.002

70. Baptista T, de Baptista EA, Ying KNM, et al. Comparative effects of the antipsychotics sulpiride or risperidone in rats. I: bodyweight, food intake, body composition, hormones and glucose tolerance. Brain Res. 2002;957:144-151. doi:10.1016/s0006-8993(02)036168

71. Goudie AJ, Smith JA, Halford JC. Characterization of olanzapineinduced weight gain in rats. J Psychopharmacol. 2002;16:291-296. doi:10.1177/026988110201600402

72. Cooper GD, Pickavance LC, Wilding JP, Harrold JA, Halford JC, Goudie AJ. Effects of olanzapine in male rats: enhanced adiposity in the absence of hyperphagia, weight gain or metabolic abnormalities. $J$ Psychopharmacol. 2007;21:405-413. doi:10.1177/ 0269881106069637

73. Pouzet B, Mow T, Kreilgaard M, Velschow S. Chronic treatment with antipsychotics in rats as a model for antipsychotic-induced weight gain in human. Pharmacol Biochem Behav. 2003;75:133140. doi:10.1016/S0091-3057(03)00042-X

74. Cooper GD, Harrold JA, Halford JC, Goudie AJ. Chronic clozapine treatment in female rats does not induce weight gain or metabolic abnormalities but enhances adiposity: implications for animal models of antipsychotic-induced weight gain. Prog Neuropsychopharmacol Biol Psychiatry. 2008;32:428-436. doi:10.1016/j.pnpbp.2007.09.012

75. Tulipano G, Rizzetti C, Bianchi I, Fanzani A, Spano P, Cocchi D. Clozapine-induced alteration of glucose homeostasis in the rat: the contribution of hypothalamic-pituitary-adrenal axis activation. Neuroendocrinology. 2007;85:61-70. doi:10.1159/000100981

76. Victoriano M, Hermier D, Even PC, et al. Early perturbation in feeding behaviour and energy homeostasy in olanzapine-treated rats. Psychopharmacology (Berl). 2009;206:167-176. doi:10.1007/ s00213-009-1593-5

77. Smith GC, Chaussade C, Vickers M, Jensen J, Shepherd PR. Atypical antipsychotic drugs induce derangements in glucose homeostasis by acutely increasing glucagon secretion and hepatic glucose output in the rat. Diabetologia. 2008;51:2309-2317. doi:10.1007/s00125-008-1152-3

78. Smith GC, Vickers MH, Cognard E, Shepherd PR. Clozapine and quetiapine acutely reduce glucagon-like peptide-1 production and increase glucagon release in obese rats: implications for glucose metabolism and food choice behaviour. Schizophr Res. 2009;115:30-40. doi:10.1016/j.schres.2009.07.011 
79. Houseknecht KL, Robertson AS, Zavadoski W, Gibbs EM, Johnson DE, Rollema H. Acute effects of atypical antipsychotics on wholebody insulin resistance in rats: implications for adverse metabolic effects. Neuropsychopharmacology. 2007;32:289-297. doi:10.1038/sj.npp.1301209

80. Chintoh AF, Mann SW, Lam L, et al. Insulin resistance and decreased glucose-stimulated insulin secretion after acute olanzapine administration. J Clin Psychopharmacol. 2008;28:494-499. doi:10.1097/ JCP.0b013e318184b4c5

81. Chintoh AF, Mann SW, Lam L, et al. Insulin resistance and secretion in vivo: effects of different antipsychotics in an animal model. Schizophr Res. 2009;108:127-133. doi:10.1016/j.schres.2008.12.012

82. Vestri HS, Maianu L, Moellering DR, Garvey WT. Atypical antipsychotic drugs directly impair insulin action in adipocytes: effects on glucose transport, lipogenesis, and antilipolysis. Neuropsychopharmacology. 2007;32:765-772. doi:10.1038/sj. npp. 1301142

83. Yang LH, Chen TM, Yu ST, Chen YH. Olanzapine induces SREBP-1-related adipogenesis in 3T3-L1 cells. Pharmacol Res. 2007;56:202-208. doi:10.1016/j.phrs.2007.05.007

84. Minet-Ringuet J, Even PC, Valet P, et al. Alterations of lipid metabolism and gene expression in rat adipocytes during chronic olanzapine treatment. Mol Psychiatry. 2007;12:562-571. doi:10.1038/sj.mp.4001948

85. Fernø J, Vik-Mo AO, Jassim G, et al. Acute clozapine exposure in vivo induces lipid accumulation and marked sequential changes in the expression of SREBP, PPAR, and LXR target genes in rat liver. Psychopharmacology (Berl). 2009;203:73-84. doi:10.1007/s00213008-1370-x

86. Raeder MB, Fernø J, Vik-Mo AO, Steen VM. SREBP activation by antipsychotic- and antidepressant-drugs in cultured human liver cells: relevance for metabolic side-effects. Mol Cell Biochem. 2006;289:167-173. doi:10.1007/s11010-006-9160-4

87. Monteiro S, Dias P, Madeira S, et al. Metabolic syndrome in dyslipidemia consultations. Rev Port Cardiol. 2006;25:821-831.

88. Yang Z, Yin JY, Gong ZC, et al. Evidence for an effect of clozapine on the regulation of fat-cell derived factors. Clin Chim Acta. 2009;408:98-104. doi:10.1016/j.cca.2009.07.021

89. Hemmrich K, Gummersbach C, Pallua N, Luckhaus C, Fehsel K. Clozapine enhances differentiation of adipocyte progenitor cells. Mol Psychiatry. 2006;11:980-981. doi:10.1038/sj.mp.4001892

90. Hemmrich K, Kappel BA, Paul NE, et al. Antipsychotic drugs increase adipose stem cell differentiation-implications for treatment with antipsychotic drugs. $J$ Clin Psychopharmacol. 2011;31:663-665. doi:10.1097/JCP.0b013e31822bf0a5

91. Sertié AL, Suzuki AM, Sertié RA, et al. Effects of antipsychotics with different weight gain liabilities on human in vitro models of adipose tissue differentiation and metabolism. Prog Neuropsychopharmacol Biol Psychiatry. 2011;35:1884-1890. doi:10.1016/j.pnpbp.2011.07.017

92. Bulua AC, Simon A, Maddipati R, et al. Mitochondrial reactive oxygen species promote production of proinflammatory cytokines and are elevated in TNFR1-associated periodic syndrome (TRAPS). J Exp Med. 2011;208:519-533. doi:10.1084/jem.20102049

93. Pillai A, Parikh V, Terry AV, Mahadik SP. Long-term antipsychotic treatments and crossover studies in rats: differential effects of typical and atypical agents on the expression of antioxidant enzymes and membrane lipid peroxidation in rat brain. $J$ Psychiatr Res. 2007;41:372-386. doi:10.1016/j.jpsychires.2006.01.011

94. Polydoro M, Schröder N, Lima MN, et al. Haloperidol- and clozapine-induced oxidative stress in the rat brain. Pharmacol Biochem Behav. 2004;78:751-756. doi:10.1016/j.pbb.2004.05.018

95. Schaffer LF, de Freitas CM, Chiapinotto CAP, et al. Harpagophytum procumbens ethyl acetate fraction reduces fluphenazine-induced vacuous chewing movements and oxidative stress in rat brain. Neurochem Res. 2016;41:1170-1184. doi:10.1007/ s11064-015-1811-y
96. Lauressergues E, Martin F, Helleboid A, et al. Overweight induced by chronic risperidone exposure is correlated with overexpression of the SREBP-1c and FAS genes in mouse liver. Naunyn Schmiedebergs Arch Pharmacol. 2011;383:423-436. doi:10.1007/ s00210-010-0597-3

97. Lauressergues E, Staels B, Valeille K, et al. Antipsychotic drug action on SREBPs-related lipogenesis and cholesterogenesis in primary rat hepatocytes. Naunyn Schmiedebergs Arch Pharmacol. 2010;381:427-439. doi:10.1007/s00210-010-0499-4

98. Canfrán-Duque A, Casado ME, Pastor O, et al. Atypical antipsychotics alter cholesterol and fatty acid metabolism in vitro. J Lipid Res. 2013;54:310-324. doi:10.1194/jlr.M026948

99. Soliman HM, Wagih HM, Algaidi SA, Hafiz AH. Histological evaluation of the role of atypical antipsychotic drugs in inducing non-alcoholic fatty liver disease in adult male albino rats (light and electron microscopic study). Folia Biol (Praha). 2013;59:173-180.

100. Bilgic S, Tastemir KD, Azirak S, Guvenc AN, Kocaman N, Ozer MK. The protective effect of thymoquinone over olanzapineinduced side effects in liver, and metabolic side effects. Bratisl Lek Listy. 2017;118:618-625. doi:10.4149/BLL_2017_119

101. Li Y, Su R, Xu S, Huang Q, Xu H. Artesunate prevents rats from the clozapine-induced hepatic steatosis and elevation in plasma triglycerides. Neuropsychiatr Dis Treat. 2017;13:2477-2487. doi:10.2147/NDT.S145069

102. Coccurello R, Moles A. Potential mechanisms of atypical antipsychotic-induced metabolic derangement: clues for understanding obesity and novel drug design. Pharmacol Ther. 2010;127:210251. doi:10.1016/j.pharmthera.2010.04.008

103. Balt SL, Galloway GP, Baggott MJ, Schwartz Z, Mendelson J. Mechanisms and genetics of antipsychotic-associated weight gain. Clin Pharmacol Ther. 2011;90:179-183. doi:10.1038/ clpt.2011.97

104. Correll CU, Malhotra AK. Pharmacogenetics of antipsychoticinduced weight gain. Psychopharmacology (Berl). 2004;174:477489. doi:10.1007/s00213-004-1949-9

105. Field BC, Chaudhri OB, Bloom SR. Obesity treatment: novel peripheral targets. $\mathrm{Br} J$ Clin Pharmacol. 2009;68:830-843. doi:10.1111/j.1365-2125.2009.03522.x

106. Gil-Campos M, Cañete RR, Gil A. Adiponectin, the missing link in insulin resistance and obesity. Clin Nutr. 2004;23:963-974. doi:10.1016/j.clnu.2004.04.010

107. Li S, Shin HJ, Ding EL, van Dam RM. Adiponectin levels and risk of type 2 diabetes: a systematic review and meta-analysis. JAMA. 2009;302:179-188. doi:10.1001/jama.2009.976

108. Sapra M, Lawson D, Iranmanesh A, Varma A. Adiposity-independent hypoadiponectinemia as a potential marker of insulin resistance and inflammation in schizophrenia patients treated with second generation antipsychotics. Schizophr Res. 2016;174:132136. doi:10.1016/j.schres.2016.04.051

109. Bartoli F, Crocamo C, Clerici M, Carrà G. Second-generation antipsychotics and adiponectin evels in schizophrenia: a comparative meta-analysis. Eur Neuropsychopharmacol. 2015;25:17671774. doi:10.1016/j.euroneuro.2015.06.011

110. Tagami K, Kashiwase Y, Yokoyama A, et al. The atypical antipsychotic, olanzapine, potentiates ghrelin-induced receptor signaling: an in vitro study with cells expressing cloned human growth hormone secretagogue receptor. Neuropeptides. 2016;58:93-101. doi:10.1016/j.npep.2015.12.010

111. Hegedűs C, Kovács D, Kiss R, et al. Effect of long-term olanzapine treatment on meal-induced insulin sensitization and on gastrointestinal peptides in female Sprague-Dawley rats. J Psychopharmacol. 2015;29:1271-1279. doi:10.1177/0269881115602952

112. Virkkunen M, Wahlbeck K, Rissanen A, Naukkarinen H, FranssilaKallunki A. Decrease of energy expenditure causes weight increase in olanzapine treatment - a case study. Pharmacopsychiatry. 2002;35:124-126. doi:10.1055/s-2002-31521 
113. Weisberg SP, McCann D, Desai M, Rosenbaum M, Leibel RL, Ferrante AW Jr. Obesity is associated with macrophage accumulation in adipose tissue. J Clin Invest. 2003;112:1796-1808. doi:10.1172/JCI19246

114. Van Gaal LF. Long-term health considerations in schizophrenia: metabolic effects and the role of abdominal adiposity. Eur Neuropsychopharmacol. 2006;16(Suppl 3):S142-S148. doi:10.1016/j.euroneuro.2006.06.005

115. Victoriano $M$, de Beaurepaire $R$, Naour $N$, et al. Olanzapineinduced accumulation of adipose tissue is associated with an inflammatory state. Brain Res. 2010;1350:167-175. doi:10.1016/j. brainres.2010.05.060

116. Devlin MJ, Yanovski SZ, Wilson GT. Obesity: what mental health professionals need to know. Am J Psychiatry. 2000;157:854-866. doi:10.1176/appi.ajp.157.6.854

117. Monji A, Kato T, Kanba S. Cytokines and schizophrenia: microglia hypothesis of schizophrenia. Psychiatry Clin Neurosci. 2009;63:257265.

118. O’Connell KE, Thakore J, Dev KK. Pro-inflammatory cytokine levels are raised in female schizophrenia patients treated with clozapine. Schizophr Res. 2014;156:1-8. doi:10.1016/j.schres.2014.03.020

119. Sobiś J, Rykaczewska-Czerwińska M, Świętochowska E, Gorczyca P. Therapeutic effect of aripiprazole in chronic schizophrenia is accompanied by anti-inflammatory activity. Pharmacol Rep. 2015;67:353-359. doi:10.1016/j.pharep.2014.09.007

120. Hatziagelaki E, Tsiavou A, Gerasimou C, et al. Effects of olanzapine on cytokine profile and brain-derived neurotrophic factor in drug-naive subjects with first-episode psychosis. Exp Ther Med. 2019;17:3071-3076. doi:10.3892/etm.2019.7285

121. Drzyzga L, Obuchowicz E, Marcinowska A, Herman ZS. Cytokines in schizophrenia and the effects of antipsychotic drugs. Brain Behav Immun. 2006;20:532-545. doi:10.1016/j.bbi.2006.02.002

122. Baker GF, Rogers HJ. Effects of psychotropic drugs on the erythrocyte permeability to glucose and ethylidene glucose. Biochem Pharmacol. 1972;21:1871-1878. doi:10.1016/0006-2952(72)90183-9

123. Ardizzone TD, Bradley RJ, Freeman AM, Dwyer DS. Inhibition of glucose transport in PC12 cells by the atypical antipsychotic drugs risperidone and clozapine, and structural analogs of clozapine. Brain Res. 2001;923:82-90. doi:10.1016/s0006-8993(01)03026-8

124. Dwyer DS, Liu Y, Bradley RJ. Dopamine receptor antagonists modulate glucose uptake in rat pheochromocytoma (PC12) cells. Neurosci Lett. 1999;274:151-154. doi:10.1016/s0304-3940(99)00712-0

125. Dwyer DS, Pinkofsky HB, Liu Y, Bradley RJ. Antipsychotic drugs affect glucose uptake and the expression of glucose transporters in PC12 cells. Prog Neuropsychopharmacol Biol Psychiatry. 1999;23:69-80.

126. Dwyer DS, Donohoe D. Induction of hyperglycemia in mice with atypical antipsychotic drugs that inhibit glucose uptake. Pharmacol Biochem Behav. 2003;75:255-260.

127. Jassim G, Skrede S, Vázquez MJ, et al. Acute effects of orexigenic antipsychotic drugs on lipid and carbohydrate metabolism in rat. Psychopharmacology (Berl). 2012;219:783-794. doi:10.1007/ s00213-011-2397-y

128. Hou JC, Pessin JE. Ins (endocytosis) and outs (exocytosis) of GLUT4 trafficking. Curr Opin Cell Biol. 2007;19:466-473. doi:10.1016/j.ceb.2007.04.018

129. Beaulieu JM, Sotnikova TD, Marion S, Lefkowitz RJ, Gainetdinov RR, Caron MG. An Akt/beta-arrestin 2/PP2A signaling complex mediates dopaminergic neurotransmission and behavior. Cell. 2005;122:261-273. doi:10.1016/j.cell.2005.05.012

130. Girault JA, Greengard P. The neurobiology of dopamine signaling. Arch Neurol. 2004;61:641-644. doi:10.1001/archneur.61.5.641

131. Martínez JA. Mitochondrial oxidative stress and inflammation: an slalom to obesity and insulin resistance. J Physiol Biochem. 2006;62:303-306.
132. Olefsky JM, Glass CK. Macrophages, inflammation, and insulin resistance. Annu Rev Physiol. 2010;72:219-246. doi:10.1146/ annurev-physiol-021909-135846

133. Baig MR, Navaira E, Escamilla MA, Raventos H, Walss-Bass C. Clozapine treatment causes oxidation of proteins involved in energy metabolism in lymphoblastoid cells: a possible mechanism for antipsychotic-induced metabolic alterations. J Psychiatr Pract. 2010;16:325-333.

134. Casademont J, Garrabou G, Miró O, et al. Neuroleptic treatment effect on mitochondrial electron transport chain: peripheral blood mononuclear cells analysis in psychotic patients. J Clin Psychopharmacol. 2007;27:284-288. doi:10.1097/JCP.0b013e318054753e

135. Ji B, La Y, Gao L, et al. A comparative proteomics analysis of rat mitochondria from the cerebral cortex and hippocampus in response to antipsychotic medications. $J$ Proteome Res. 2009;8:3633-3641. doi:10.1021/pr800876z

136. Kotzka J, Müller-Wieland D. Sterol regulatory element-binding protein (SREBP)-1: gene regulatory target for insulin resistance. Expert Opin Ther Targets. 2004;8:141-149. doi:10.1517/14728222.8.2.141

137. Shimano H. Sterol regulatory element-binding proteins (SREBPs): transcriptional regulators of lipid synthetic genes. Prog Lipid Res. 2001;40:439-452.

138. Fernø J, Raeder MB, Vik-Mo AO, et al. Antipsychotic drugs activate SREBP-regulated expression of lipid biosynthetic genes in cultured human glioma cells: a novel mechanism of action. Pharmacogenomics J. 2005;5:298-304. doi:10.1038/sj.tpj.6500323

139. Cai HL, Tan QY, Jiang P, et al. A potential mechanism underlying atypical antipsychotics-induced lipid disturbances. Transl Psychiatry. 2015;5:e661. doi:10.1038/tp.2015.161

140. McKenney JM, Sica D. Prescription omega-3 fatty acids for the treatment of hypertriglyceridemia. Am J Health Syst Pharm. 2007;64:595-605. doi:10.2146/ajhp060164

141. Hassanali Z, Ametaj BN, Field CJ, Proctor SD, Vine DF. Dietary supplementation of n-3 PUFA reduces weight gain and improves postprandial lipaemia and the associated inflammatory response in the obese JCR:LA-cp rat. Diabetes Obes Metab. 2010;12:139-147. doi:10.1111/j.1463-1326.2009.01130.x

142. Pachikian BD, Neyrinck AM, Cani PD, et al. Hepatic steatosis in n3 fatty acid depleted mice: focus on metabolic alterations related to tissue fatty acid composition. BMC Physiol. 2008;8:21. doi:10.1186/1472-6793-8-21

143. Pachikian BD, Essaghir A, Demoulin JB, et al. Hepatic n-3 polyunsaturated fatty acid depletion promotes steatosis and insulin resistance in mice: genomic analysis of cellular targets. PLoS One. 2011;6:e23365. doi:10.1371/journal.pone.0023365

144. Qi K, Fan C, Jiang J, et al. Omega-3 fatty acid containing diets decrease plasma triglyceride concentrations in mice by reducing endogenous triglyceride synthesis and enhancing the blood clearance of triglyceride-rich particles. Clin Nutr. 2008;27:424-430. doi:10.1016/j.clnu.2008.02.001

145. Araya J, Rodrigo R, Videla LA, et al. Increase in long-chain polyunsaturated fatty acid $n-6 / n-3$ ratio in relation to hepatic steatosis in patients with non-alcoholic fatty liver disease. Clin Sci (Lond). 2004;106:635-643. doi:10.1042/CS20030326

146. Burrows T, Collins CE, Garg ML. Omega-3 index, obesity and insulin resistance in children. Int J Pediatr Obes. 2011;6:e532e539. doi:10.3109/17477166.2010.549489

147. Evans DR, Parikh VV, Khan MM, Coussons C, Buckley PF, Mahadik SP. Red blood cell membrane essential fatty acid metabolism in early psychotic patients following antipsychotic drug treatment. Prostaglandins Leukot Essent Fatty Acids. 2003;69:393-399.

148. Ranjekar PK, Hinge A, Hegde MV, et al. Decreased antioxidant enzymes and membrane essential polyunsaturated fatty acids in schizophrenic and bipolar mood disorder patients. Psychiatry Res. 2003;121:109-122. 
149. Reddy RD, Keshavan MS, Yao JK. Reduced red blood cell membrane essential polyunsaturated fatty acids in first episode schizophrenia at neuroleptic-naive baseline. Schizophr Bull. 2004;30:901911. doi:10.1093/oxfordjournals.schbul.a007140

150. Caniato RN, Alvarenga ME, Garcia-Alcaraz MA. Effect of omega-3 fatty acids on the lipid profile of patients taking clozapine. Aust N Z J Psychiatry. 2006;40:691-697. doi:10.1080/ j.1440-1614.2006.01869.x
151. Peet M, Horrobin DF. A dose-ranging exploratory study of the effects of ethyl- eicosapentaenoate in patients with persistent schizophrenic symptoms. J Psychiatr Res. 2002;36:7-18.

152. McNamara RK, Magrisso IJ, Hofacer R, et al. Omega-3 fatty acid deficiency augments risperidone-induced hepatic steatosis in rats: positive association with stearoyl-CoA desaturase. Pharmacol Res. 2012;66:283-291. doi:10.1016/j.phrs.2012.06.010

\section{Publish your work in this journal}

Neuropsychiatric Disease and Treatment is an international, peerreviewed journal of clinical therapeutics and pharmacology focusing on concise rapid reporting of clinical or pre-clinical studies on a range of neuropsychiatric and neurological disorders. This journal is indexed on PubMed Central, the 'PsycINFO' database and CAS, and is the official journal of The International Neuropsychiatric Association (INA). The manuscript management system is completely online and includes a very quick and fair peer-review system, which is all easy to use. Visit http://www.dovepress.com/testimonials.php to read real quotes from published authors. 